\title{
The Role of RhoA in Neovascular-Related Functions of Endothelial Progenitor Cells Induced by Angiotensin II
}

\author{
Jin-Xiu Yanga, ban-Yun Pan ${ }^{a}$ Bin Chen ${ }^{c}$ Yuan-Gang Qiu ${ }^{a}$ Wei Mao \\ Shen-Jiang $\mathrm{Hu}^{\mathrm{b}}$ \\ aDepartment of Cardiology, the First Affiliated Hospital of Zhejiang Chinese Medical University, \\ Hangzhou, 'Institute of Cardiology, the First Affiliated Hospital, School of Medicine, Zhejiang University, \\ Hangzhou, 'Department of Cardiology, Hangzhou Peoples Hospital 1, Nanjing Medical University, \\ Hangzhou, P.R. China
}

\section{Key Words}

Endothelial progenitor cell $\bullet$ Neovascular-related functions $\bullet$ RhoA/ROCK $\bullet$ Signaling pathway

\begin{abstract}
Background/Aims: Interference with endothelial progenitor cell (EPC) neovascularization is a novel therapeutic target for neovascular-related diseases. Angiotensin II (Ang II) was found to enhance new vessel formation and aggravated neovascular-related diseases. In this study, we investigated the effects of Ang II on EPC neovascular-related functions and explored the underlying mechanisms. Methods: EPCs were cultured from bone marrow derived mononuclear cells. The effects of Ang II on EPC proliferation, adhesion, migration, and in vitro tube formation were investigated using the MTT assay, adhesion assay, transwell chamber assay, and in vitro tube formation assay respectively. The underlying mechanisms were explored using Western blotting assay. Results: EPC adhesion, migration and in vitro tube formation were promoted by Ang II, and the effects were reversed by RhoA/Rho-associated kinases (ROCK) signaling pathway inhibitors including C3 exoenzyme, GGTI-286 and Y-27632. The active form of RhoA was up-regulated by Ang II and this effect was abolished by C3 exoenzyme. Moreover, RhoA silencing resulted in a notable inhibition of EPC adhesion, migration and in vitro tube formation, suggesting that RhoA activation played a pivotal role in Ang II angiogenic effect. The results also demonstrated that phosphorylation of p38 mitogen-activated protein kinase (MAPK) and c-Jun-NH2 kinase was elevated by Ang II and attenuated by C3 exoenzyme, GGTI-286 and Y-27632. The enhancing effects of Ang II on EPC adhesion, migration and in vitro vasculogenesis were reversed by p38 inhibitor SB202190 and JNK inhibitor SP600125. Conclusion: Ang II may enhance EPC neovascular-related functions through activating RhoA/ ROCK and MAPK signaling pathway.
\end{abstract}




\section{Introduction}

Neovascularization is involved in the pathogenesis of cancer, diabetic retinopathy, and atherosclerosis $[1,2]$. Furthermore, neovascularization may play a role in the treatment of peripheral vascular and coronary artery disease [3]. The regulation of neovascularization may represent an important therapeutic target for neovascular-related diseases. Recent studies have indicated that endothelial progenitor cells (EPCs) contribute to neovascularization [2].

EPCs comprise a group of cells that have the capacity to circulate, proliferate and differentiate into mature endothelial cells (ECs) but that have not yet acquired characteristic mature endothelial markers nor formed a lumen [4]. These precursor cells not only participate in angiogenesis, but also contribute to postnatal vasculogenesis [4, 5]. Interference with EPC neovascularization may be a novel therapeutic strategy in the treatment of neovascularrelated diseases.

Activation of the renin-angiotensin system (RAS) is associated with many neovascularrelated diseases [6]. Angiotensin II (Ang II ) is an important biologically active component of the RAS. Emerging evidence suggested that Ang II increases vessel density and enhances angiogenesis [7], thus may aggravate the process of neovascular-related diseases. In this paper, we studied the effects of Ang II on EPC neovascular-related functions including proliferation, adhesion, migration, and in vitro tube formation. Our previous study indicated that RhoA and its effector protein Rho-associated kinases (ROCK) may play a role in the effects of Zoledronate on EPC neovascular-related functions [2]. However, the role of RhoA/ ROCK and its following signaling pathways on EPC neovascular-related functions was not systematically explored in previous studies. So, we also attempted to find out whether the modulation is RhoA/ROCK signaling dependent.

\section{Materials and Methods}

\section{Cell culture}

All animal investigations were conducted in accordance with the Guide for the Care and Use of Laboratory Animals published by NIH. Male Sprague-Dawley rats of 6 to 7 weeks old aa were fed with conventional diet.

In vitro expansion of rat bone marrow-derived EPCs was performed as we previously described $[2,8,9]$. Briefly, the mononuclear cells (MNCs) fraction was obtained by density gradient centrifugation from the femurs of male rats. Cells were then suspended in EBM-2 medium (Lonza) supplemented with 10\% FBS (Gibco) and plated on 6-well plates (Corning). After $24 \mathrm{~h}$ and $48 \mathrm{~h}$, the non-adherent cells were aspirated and transferred to new plates. Only the non-adherent cells harvested after $48 \mathrm{~h}$ were cultured in EBM-2 medium supplemented with EGM-2 MV single aliquots containing 10\% FBS, vascular endothelial growth factor (VEGF), epidermal growth factor, fibroblast growth factor-2, insulin-like growth factor-1, and ascorbic acid. Non-adherent cells were removed by washing after $4 \mathrm{~d}$ in culture and new media was applied every 3 days.

\section{EPC fluorescent staining}

Fluorescent chemical detection of EPCs was performed on attached MNCs after $14 \mathrm{~d}$ in culture. Direct fluorescent staining was used to detect dual binding of 1,1-dioctadecyl-3, 3, 3, 3-tetramethylindocarbocyanine (DiI)-labeled acetylated low-density lipoprotein (acLDL; Molecular Probe) and fluorescein isothiocyanate (FITC)-conjugated Ulex europaeus agglutinin (UEA)-I (Sigma). The cells were first incubated with acLDL $(2.4 \mu \mathrm{g} / \mathrm{ml})$ at $37^{\circ} \mathrm{C}$ and later fixed with $2 \%$ paraformaldehyde for $10 \mathrm{~min}$. After washing, EPCs were reacted with UEA-I $(10 \mu \mathrm{g} / \mathrm{ml})$ for $1 \mathrm{~h}$. After staining, samples were viewed with fluorescence microscopy $(\times 200)$. Fluorescence microscopy identified double-positive cells as EPCs.

\section{KARGER}




\section{Cellular Physiology Cell Physiol Biochem 2018;47:2498-2510 \\ \begin{tabular}{c|c} 
DOI: 10.1159/000491623 & O 2018 The Author(s). Published by S. Karger AG, Basel \\
www.karger.com/cpb
\end{tabular}}

Yang et al.:The Role of RhoA in Neovascular-Related Functions of EPCS

Flow cytometry cell analysis

To identify EPCs, the cells were trypsinized, followed by repeated gentle flushing through a pipette tip. Cells $\left(2 \times 10^{5}\right)$ were incubated for $30 \mathrm{~min}$ at $4^{\circ} \mathrm{C}$ with anti-vascular endothelial growth factor receptor 2 (anti-VEGFR2, Abcam) and phycoerythrinconjugated monoclonal antibodies against CD31 (R\&D), and von Willebrand factor (vWF, Abcam). A FITC-conjugated antimouse antibody (Vector) was added for staining with VEGFR2. Isotypeidentical antibodies served as controls. Flow cytometric analyses were performed by using a FACStar flow cytometer (Beckman Coulter).

\section{Cell proliferation assay}

EPC proliferation was evaluated using a standard 3-(4, 5-dimethylthiazol-2-yl)-2, 5-diphenyltetrazolium bromide (MTT) assay. After being cultured for $7 \mathrm{~d}$, cells were digested with $0.25 \%$ trypsin and then cultured in EBM-2 medium containing 10\% FBS in 96-well culture plate $(200 \mu \mathrm{l} /$ well $)$. After being cultured for $48 \mathrm{~h}$, the supernatant was then discarded by aspiration and serum-free EBM-2 medium was added. Ang II $(0,0.1,1,10$, and $100 \mu \mathrm{M})$ was added, respectively ( $200 \mu \mathrm{l} /$ well). In order to test the effects of RhoA/ROCK and mitogenactivated protein kinase (MAPK) pathway inhibitors on cell proliferation, C3 exoenzyme (30 $\mathrm{ng} / \mathrm{mL})$, GGTI-286 (10 $\mu \mathrm{M}), \mathrm{Y}-27632(10 \mu \mathrm{M}), \mathrm{SB} 202190(10 \mathrm{mmol} / \mathrm{L})$, or SP600125 (10 $\mathrm{mmol} / \mathrm{L}$ ) was added respectively. After being incubated for $30 \mathrm{~min}$, EPCs of each well were treated with Ang II $(1 \mu \mathrm{M})$. After being incubated for $48 \mathrm{~h}$, they were supplemented with $20 \mu \mathrm{l}$ MTT (5 g/l, Fluka Co. Product) and incubated for another $4 \mathrm{~h}$. The supernatant was aspirated and the EPCs preparation was shaked with $150 \mu \mathrm{l}$ dimethyl sulfoxide (DMSO) for $10 \mathrm{~min}$, before the OD value was measured at $490 \mathrm{~nm}$.

\section{EPC migration assay}

EPC migration was evaluated by using a transwell chamber (Greiner) with 8- $\mu$ m pore filters. In brief, isolated cells were detached using $0.25 \%$ trypsin, harvested by centrifugation, resuspended in $500 \mu \mathrm{l} \mathrm{EBM}-2$ medium and counted, then $2 \times 10^{4}$ EPCs were placed in the upper chamber of a transwell chamber. VEGF in serum-free EBM-2 medium was placed in the lower compartment of the chamber. After $24 \mathrm{~h}$ incubation at $37^{\circ} \mathrm{C}$ in $5 \%$ humidified $\mathrm{CO}_{2^{\prime}}$, the lower side of the filter was washed with PBS and cells remaining on the upper face were removed with a cotton wool swab. Transwell filters were fixed with $2 \%$ paraformaldehyde. For quantification, EPCs were stained with $0.1 \%$ crystal violet solution. Cells migrating into the lower chamber were counted in five random microscopic fields $(\times 200)$.

\section{Adhesion assay}

After incubating with Ang II and C3 exoenzyme, GGTI-286, Y-27632, SB202190, SP600125 respectively for $48 \mathrm{~h}$, EPCs were washed with PBS and gently detached with $0.25 \%$ trypsin. After centrifugation and resuspension in EBM-2 medium, identical cells were replated onto fibronectin-coated culture dishes and incubated for $30 \mathrm{~min}$ at $37^{\circ} \mathrm{C}$ in $5 \%$ humidified $\mathrm{CO}_{2}$. After incubation, non-adherent cells were removed by washing with PBS twice. Adherent cells were counted by independent blinded investigators in five randomly chosen microscopic fields $(\times 400)$.

In vitro tube formation assay

ECMatrixTM solution (Chemicon) was thawed on ice overnight, mixed with $10 \times$ ECMatrixTM diluent and placed in a 96 -well plate at $37^{\circ} \mathrm{C}$ for $1 \mathrm{~h}$ to allow the matrix solution to solidify. Then EPCs $\left(2 \times 10^{4}\right.$ cells $)$ were harvested and replated on top of the solidified matrix solution with $150 \mu \mathrm{l} \mathrm{EBM}-2$ medium. Cells were incubated at $37^{\circ} \mathrm{C}$ for 18 $\mathrm{h}$ and fixed with $2 \%$ paraformaldehyde. The lengths of enclosed tubes within the network were measured from five random microscopic fields $(\times 200)$.

Lentivirus vectors for RhoA small hairpin RNA and cell infection

A third generation self-inactivating lentivirus vector containing a CMV-driven GFP reporter and a U6 promoter upstream of the cloning restriction sites (BamHI and EcoRI) 


\section{Cellular Physiology Cell Physiol Biochem 2018;47:2498-2510 \begin{tabular}{l|l} 
DOI: 10.1159/000491623 & O 2018 The Author(s). Published by S. Karger AG, Basel \\
www.karger.com/cpb
\end{tabular} \\ Yang et al.:The Role of RhoA in Neovascular-Related Functions of EPCS}

was used. Four coding regions corresponding to targeting rat RhoA starting at positions 153, 81, 354 and 40 in the sequence (GenBank Accession: NM_057132.3) were selected as siRNA target sequences under the guide of siRNA designing software offered by GenePharma Co., Ltd, Shanghai, China. Four shRNA-RhoA lentivirus vectors were respectively constructed by Genepharma. The Lv-NC-shRNA which included the GFP gene was designed with a randomly chosen nonsense sequence to serve as negative control. The most active shRNA against the rat RhoA target DNA sequence, 5'-GATCCGCAGGTAGAGTTGGCTTTATGTTCAAGAGA CATAAAGCCAACTCTACCTGCTTTTTTG-3', which starting at position 153 was selected. Recombinant lentivirus vectors were produced by co-transfecting $293 \mathrm{~T}$ cells with the lentivirus expression plasmid and packaging plasmids. The virus titers produced were approximately $10^{9}$ transducing $\mathrm{U} / \mathrm{ml}$ medium.

When the cells were about $80 \%$ confluent in EBM-2 medium, they were detached and subcultured at $1 \times 10^{6}$ cells per well into six-well plates. After $24 \mathrm{~h}$ culture, cells were infected with recombinant lentivirus vectors at a multiplicity of infection (MOI) of 100. Polybrene was added to each well at a concentration of $5 \mathrm{mg} / \mathrm{ml}$.

RhoA activity assay

RhoA activity was determined by pull-down assay using a Rho activation assay kit (Cytoskeleton). EPCs were placed in serum-free EBM-2 medium for $12 \mathrm{~h}$ and stimulated with $1 \mu \mathrm{M}$ Ang II for $15 \mathrm{~min}$ at $37^{\circ} \mathrm{C}$. C3 Exoenzyme (30 ng/mL) was added $48 \mathrm{~h}$ before stimulation. Cells were then lyzed with lysis buffer and a protein assay was performed prior to the pull-down assay to equalize total protein concentration in each treated group. Whole cell lysates were incubated with agarose-conjugated rhotekin-RBD at $4^{\circ} \mathrm{C}$ for $60 \mathrm{~min}$ and then washed twice with wash buffer. Agarose beads were boiled in SDS-PAGE sample buffer to release active Rho prior to undergoing precipitation with the Rhotekin GTP-Rho. After precipitation bound Rho proteins were detected by Western blotting using a specific antiRhoA antibody (Cytoskeleton). Meanwhile, $20 \mu \mathrm{g}$ total cell lysate per sample was used to detect the total amount of RhoA.

\section{Western blotting assay}

The protein contents of the cell lysates were determined using a micro BCA kit (Beyotime). Proteins from cell lysates were mixed with $4 \times$ loading buffer (Invitrogen) and boiled for 10 minutes, before electrophoresis on $10 \%$ sodium dodecyl sulfate-polyacrylamide gels. Following transfer onto polyvinylidene fluoride membranes and blocking, membranes were incubated with antibodies against RhoA, phospho-p38, p38, phospho-JNK, JNK, phospho-ERK1/2 or ERK1/2 (1:1000, Cell Signaling). Following three washes in TBST, membranes were subsequently incubated with horseradish peroxidase-conjugated goat anti-rabbit IgG antibody (1:3000, Sigma-Aldrich). The signals were detected by enhanced chemiluminescence reagents (Thermo) and exposure to X-ray film. The density of the bands was quantified by using Image J software (National Institutes of Health). Expression of RBD binding RhoA was normalized to total RhoA expression in the same lane and phospho-p38, JNK, ERK1/2 were normalized to total p38, JNK and ERK1/2 respectively.

\section{Statistical analysis}

All data are presented as mean \pm SD. Differences between group means were assessed by ANOVA for multiple comparisons using SPSS 16.0. Values of $\mathrm{P}<0.05$ were considered significant.

\section{Results}

Characterization of EPCS

Total MNCs isolated and cultured for $14 \mathrm{~d}$ resulted in a spindle-shaped, ECs-like morphology (Fig. 1A). EPCs were characterized as adherent cells double positive for DiLDL 


\section{Cellular Physiology and Biochemistry}

Fig. 1. Immunofluorescence identification and immunophenotype of bone marrow derived-EPCs. The attached cells exhibited a spindle shaped, endothelial cells like morphology (A), and adherent cells DiLDL uptake (B: red, exciting wave-length $543 \mathrm{~nm}$ ) and lectin binding ( $\mathrm{C}$ : green, exciting wave-length $477 \mathrm{~nm}$ ) were assessed under a fluorescence microscopy. Double positive cells appearing yellow in the overlay (D) were identified as differentiating EPCs $(\times 200)$. E to $G$, Membrane antigen expression on bone marrow-derived EPCs was determined by flow cytometry. Scale bar, 100 $\mu \mathrm{m}$.

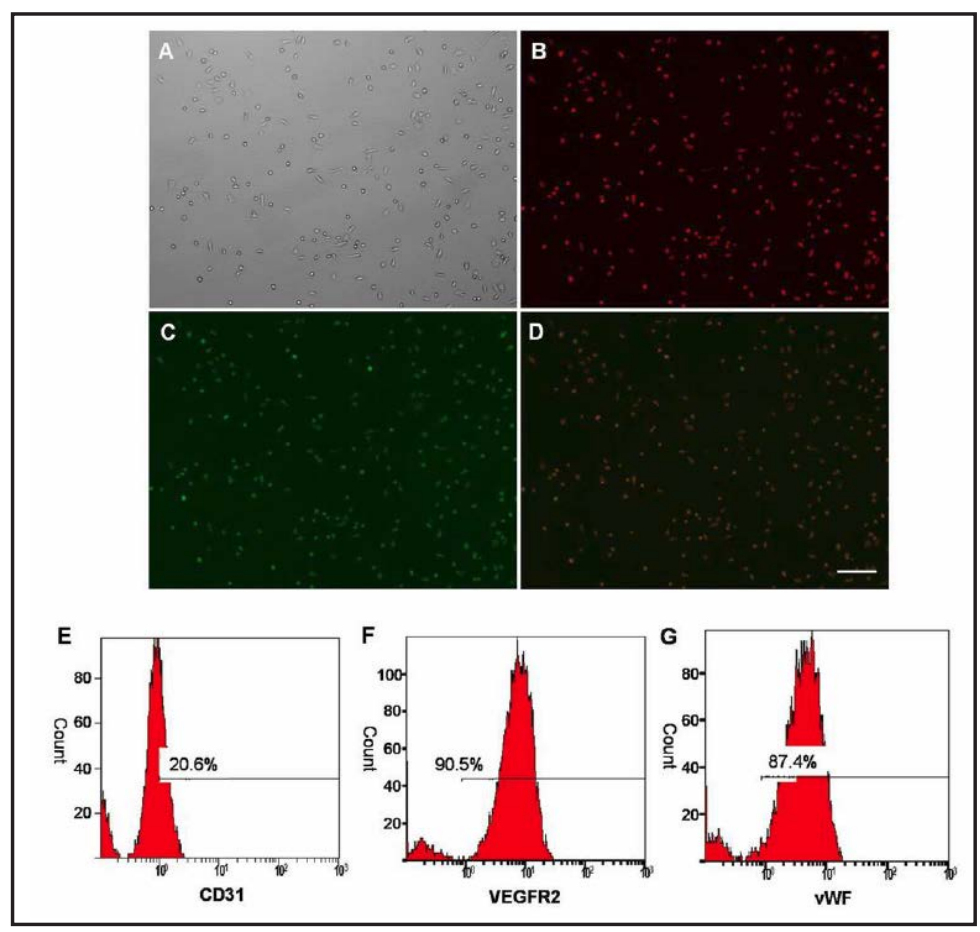

uptake and lectin binding by using fluorescence microscopy (Fig. 1B-D). These cells were characterized further by demonstrating the expression of CD31, VEGFR2 and vWF by flow cytometry (Fig. 1E-G).

\section{Effect of Ang II on the proliferation of EPCS}

EPC proliferation was assayed using the MTT assay. Ang II had no effect on EPC proliferation (data not shown). When incubated with C3 exoenzyme, GGTI-286, Y-27632, SB202190, or SP600125 for 48 h, cell viability did not show marked changes compared with control group (data not shown).

\section{Effect of Ang II on EPC adhesion}

To assess the effects of Ang II on cell adhesion, EPCs were treated with C3 exoenzyme, GGTI-286, Y-27632, SB202190, or SP600125 for 30 min and then with indicated concentration of Ang II for $48 \mathrm{~h}$. After replating on fibronectin-coated dishes, EPCs pre-exposed to Ang II exhibited a significant increase in the number of adhesive cells, which became apparent at 1 and $10 \mu \mathrm{M}(\mathrm{P}<0.05)$ (Fig. 2Aa). EPC adhesion ability was attenuated by C3 exoenzyme, GGTI-286 and Y-27632 (Fig. $2 \mathrm{Ab}$ ). The result also showed that EPC adhesion ability was decreased by SB202190 and SP600125 (Fig. 4B). In addition, C3 alone, GGTI-286 alone, or Y27632 alone had no effect on EPC adhesion ability (Fig. 5A).

\section{Effect of Ang II on EPC migration}

EPC migration was analyzed by the transwell chamber assay. The result showed that Ang II promoted EPC migration, which became apparent at 1 and $10 \mu \mathrm{M}$ (Fig. 2Ba). EPC migration capacity was impaired by C3 exoenzyme, GGTI-286 and Y-27632 (Fig. 2Bb and 2Bc). It was also found that EPC migration was attenuated by SB202190 and SP600125 (Fig. 4A). In addition, C3 alone, GGTI-286 alone, or Y27632 alone had no effect on EPC migration ability (Fig. 5B).

Effect of Ang II on tube formation of EPCs in vitro

EPCs were incubated overnight in starvation medium and then stimulated for $48 \mathrm{~h}$ with indicated treatment in basal medium. The lengths of enclosed tubes which formed 
Fig. 2. Neovascular-related functions of EPCs treated with Ang II and RhoA/ROCK signaling inhibitors. (A): Adhesion capacity of EPCs treated with Ang II and RhoA/ ROCK signaling inhibitors. Adhesion capacity of EPCs was promoted by Ang II and this effect could be reversed by C3 exoenzyme, GGTI-286 and Y-27632. Data are presented as mean $\pm S D, n=5$. ${ }^{*} \mathrm{P}<0.05$ vs. control group; ${ }^{~} \mathrm{P}<0.05$ vs. Ang II group; ** $\mathrm{P}<0.01$ vs. Ang II group; (B): Migration of EPCs cocultured with Ang II and RhoA/ROCK signaling inhibitors. Migratory ability of EPCs was enhanced by Ang II and this effect could be reversed by C3 exoenzyme, GGTI-286 and Y- 27632. Data are presented as mean $\pm \mathrm{SD}, \mathrm{n}=5$. $\mathrm{P}<0.05$ vs. control group; ${ }^{* *} \mathrm{P}<0.01$ vs. Ang II group; (C): Effect of Ang II and RhoA/ROCK signaling inhibitors on EPC in vitro vasculogenesis. EPC in vitro vasculogenesis function was increased by Ang II and this effect could be reversed by $\mathrm{C} 3$ exoenzyme, GGTI-286 and Y-27632. Data are presented as mean $\pm \mathrm{SD}, \mathrm{n}=5$. * $\mathrm{P}<0.05$ vs. control group; ${ }^{* *} \mathrm{P}<0.01$ vs. control group; ${ }^{*} \mathrm{P}<0.05$ vs. Ang II group; ${ }^{\&} \mathrm{P}<0.01$ vs. Ang II group.
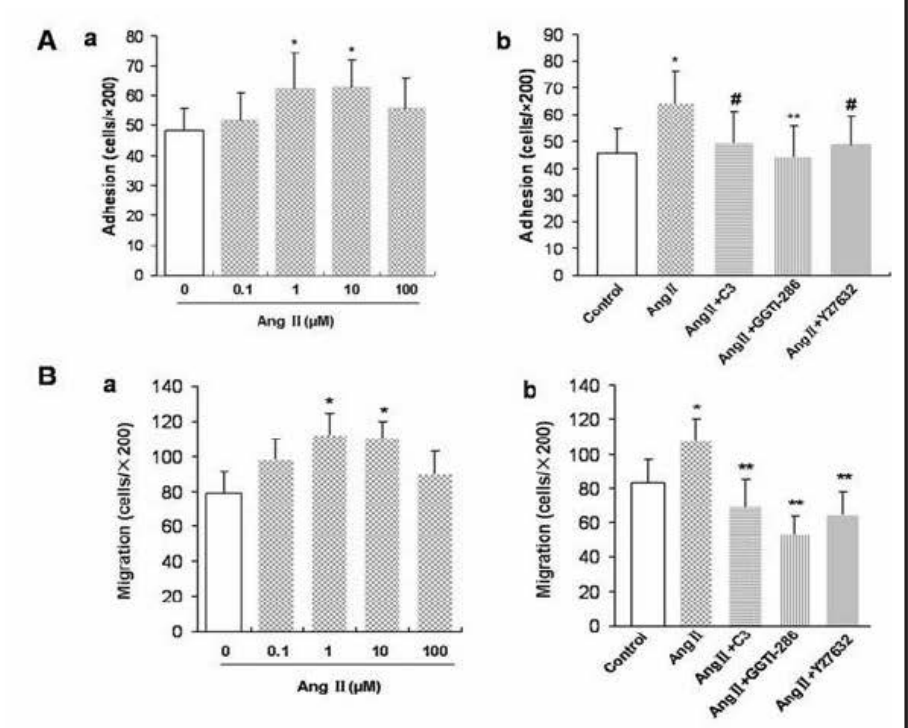

c
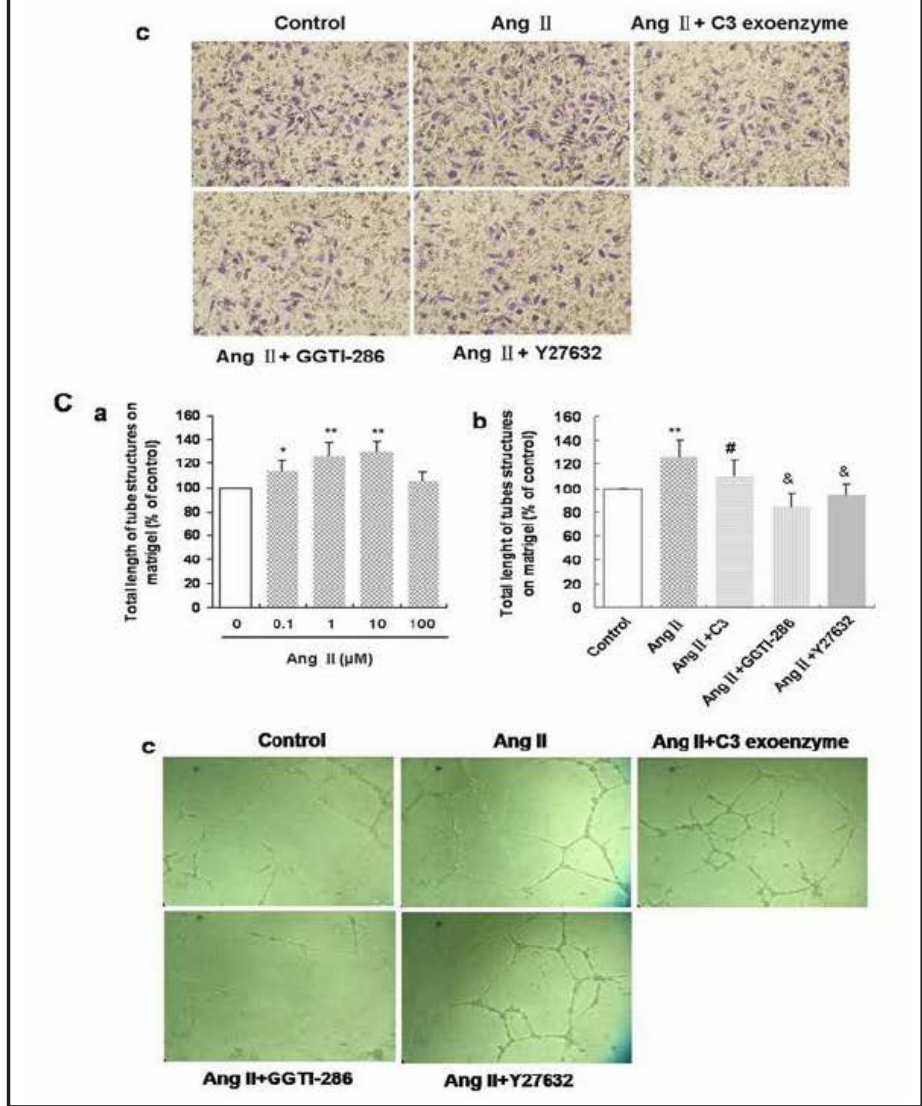

Ang II

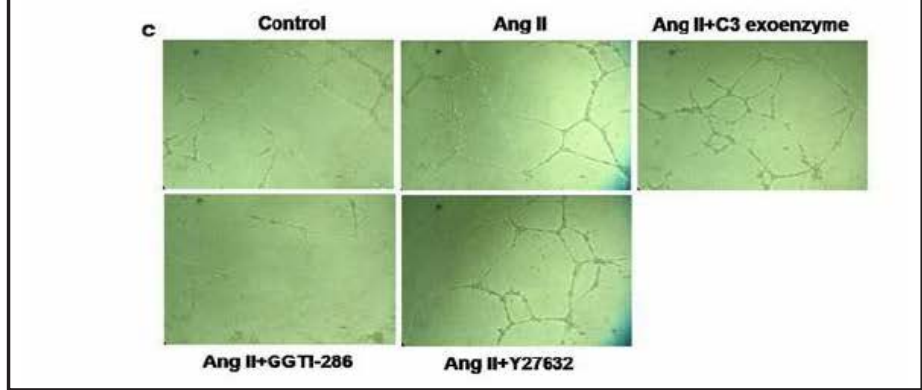

within the network were measured. As shown in Fig. 2Ca, Ang II significantly promoted EPC vasculogenesis in vitro, which became apparent at $0.1 \mu \mathrm{M}$, with a peak at $10 \mu \mathrm{M}$. C3 exoenzyme, GGTI-286 and Y-27632 decreased in vitro tube formation ability of EPCs (Fig. $2 \mathrm{Cb}$ and $2 \mathrm{Cc}$ ). The result also showed that EPC in vitro tube formation ability was impaired by SB202190 and SP600125 (Fig. 4C). In addition, C3 alone, GGTI-286 alone, or Y27632 alone had no effect on EPC in vitro tube formation ability (Fig. 5C). 
A

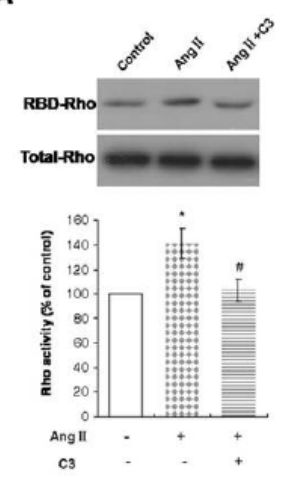

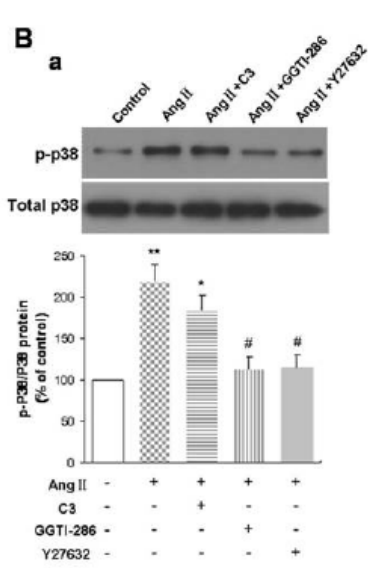

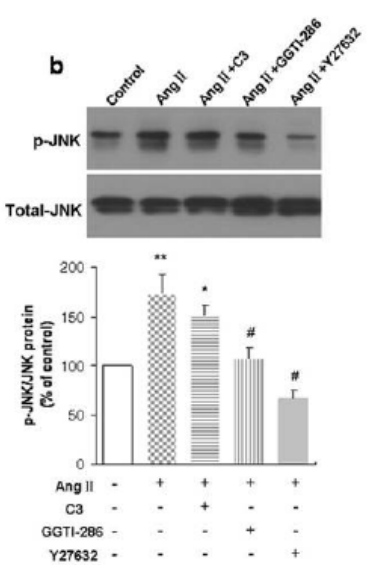

Fig. 3. The role of RhoA/ROCK and MAPK signaling pathway in neovascular-related functions of EPCs treated with Ang II . (A): Effect of Ang II and C3 exoenzyme on EPC RhoA activation. Serum-starved EPCs were cocultured with or without C3 exoenzyme before incubation with Ang II for $15 \mathrm{~min}$. Bound RhoA proteins were detected by Western blot using monoclonal antibody against RhoA (upper panel). Western blotting of the total amount of RhoA in cell lysates (lower panel) was also performed in the same lysates. The results are representative of three independent experiments. ${ }^{*} \mathrm{P}<0.01$ vs. control group; ${ }^{\#} \mathrm{P}<0.01$ vs. Ang II group; (B): Involvement of p38 (a) and JNK (b) signaling in Ang II , C3 exoenzyme, GGTI-286, and Y-27632 effects on EPCs. Values shown are representative of three independent experiments. ${ }^{* *} \mathrm{P}<0.01$ vs. control group; * $\mathrm{P}<0.05$ vs. Ang II group; ${ }^{\mathrm{P}}<0.01$ vs. Ang II group.

Effect of Ang II on RhoA activation of EPCS

A pull-down assay with the fusion protein GST-RBD, which specifically recognizes RhoGTP, the active form of Rho, was used to investigate the effect of Ang II on RhoA activation of EPCs. The active form of RhoA (GTP-bound) increased in EPCs treated with Ang II for $15 \mathrm{~min}$ (Fig. 3A). Pretreatment with C3 exoenzyme for 30 min markedly reduced RhoA activation (Fig. 3A). These results suggest that RhoA activation is promoted by Ang II and attenuated by $\mathrm{C} 3$ exoenzyme.

RhoA activation played a pivotal role in Ang II angiogenic effect

To explore the role of RhoA signaling in the angiogenic effect of Ang II , we used shRNA to silence RhoA gene expression and investigated its effect on EPC migration, adhesion and in vitro vasculogenesis capacities. The data showed that the expression of active form of RhoA was decreased markedly in RhoA shRNA interference group compared with control shRNA interference group ( $\mathrm{P}<0.01$, Fig. $6 \mathrm{~A}$ ). Then we investigated the role of RhoA gene silence in the effect of Ang II on EPC angiogenic property. The results showed that EPC migration, adhesion and in vitro vasculogenesis capacities were promoted by Ang II stimulation. Meanwhile, RhoA silencing resulted in a notable inhibition of EPC migration, adhesion and in vitro vasculogenesis capacities $(\mathrm{P}<0.05$, Fig. 6B-D). These results suggested that RhoA activation played a pivotal role in Ang II angiogenic effect.

\section{Effect of Ang II on MAPK activation of EPCs}

The amount of p-38 phosphorylation was promoted to $218.31 \pm 21.48 \%$ of control after administration of $1 \mu \mathrm{M}$ Ang II $(\mathrm{P}<0.01$, Fig. 3Ba). The phosphorylation of $\mathrm{p}-38$ was markedly decreased in EPCs that pretreated with C3 exoenzyme, GGTI-286, and Y-27632 (Fig. 3Ba). The result also showed that JNK phosphorylation was increased to $172.89 \pm 19.24$ $\%$ of control after administration of $1 \mu \mathrm{M}$ Ang II $(\mathrm{P}<0.01$, Fig. 3Bb). The phosphorylation of JNK was markedly reduced in EPCs that pretreated with C3 exoenzyme, GGTI-286 and Y-27632 (Fig. 3Bb). The phosphorylation of p-38 and JNK could be significantly reduced by SB202190 and SP600125 respectively (Fig. 4D). Neither total p-38 nor JNK was changed. 
Fig. 4. Effect of p38 and JNK inhibitors on the functions of EPCs induced by Ang II. EPC adhesion, migration and in vitro vasculogenesis capacities were enhanced by Ang II and these effects could be reversed by p38 inhibitor SB202190 and JNK inhibitor SP600125 (AC). Data are presented as mean $\pm \mathrm{SD}, \mathrm{n}=5$. \& $\mathrm{P}<0.05$ vs. control group; \# $\mathrm{P}<0.01$ vs. control group; * $\mathrm{P}<$ 0.05 vs. Ang II group; ** $\mathrm{P}<0.01$ vs. Ang II group. SB202190 inhibited p38 activation while SP600125 inhibited JNK activation in EPCs induced by Ang II (D). Data are presented as mean \pm $\mathrm{SD}, \mathrm{n}=3$. ${ }^{\#} \mathrm{P}<0.01$ vs. control group; ** $\mathrm{P}<$ 0.01 vs. Ang II group.

\section{A}

a

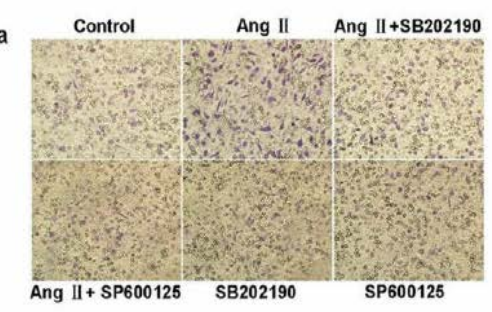

b

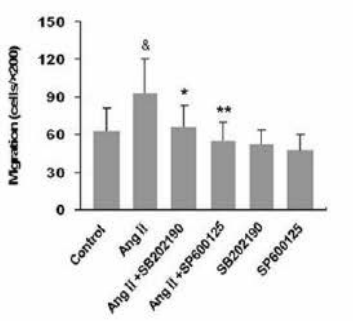

c a

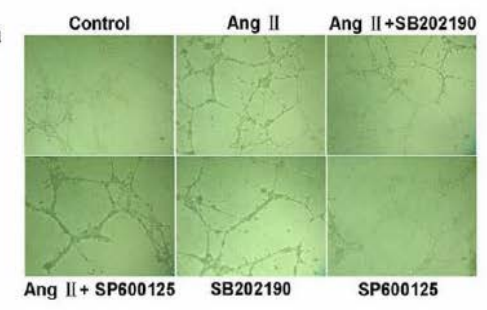

b

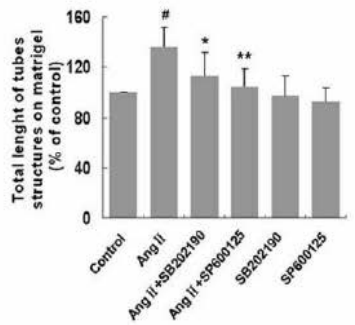

B

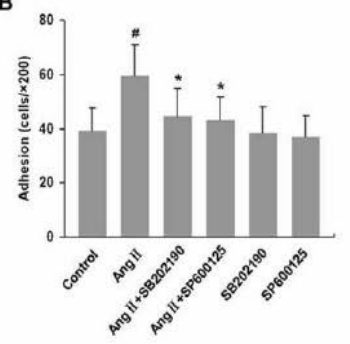

D
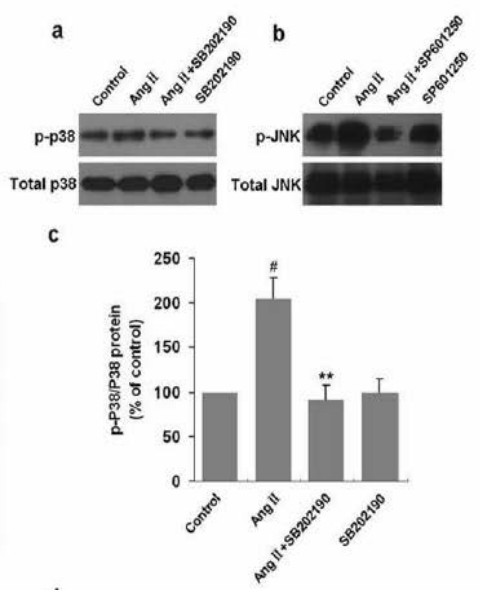

d

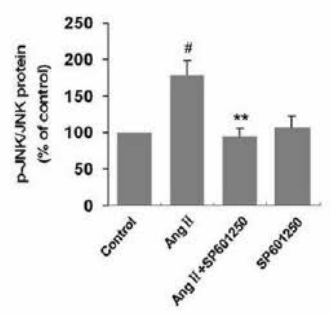

Ang II had no obvious influence on Erk1/2 phosphorylation (data not shown). These results suggest the involvement of p-38 and JNK signal pathway in the role of Ang II effects on EPCs.

\section{Discussion}

Numerous clinical and experimental studies have demonstrated that RAS is critically involved in the development of cardiovascular diseases [10]. Pharmacotherapeutical modulation of the RAS is one of the most important treatment modalities in neovascularrelated diseases. Recent studies have showed that EPCs take part in vasculogenesis and angiogenesis $[4,5]$. EPCs are served as a novel target for the treatment of neovascularrelated diseases.

In the study, we investigated the effects of Ang II on EPC neovascular-related functions. The findings showed that EPC proliferation was not affected by Ang II while adhesion and migration were promoted by Ang II. The observation that Ang II affected EPC adhesion and migration prompted us to investigate the possibility that Ang II could also enhance angiogenesis. The results of in vitro tube formation assay showed that EPC in vitro tube formation was profoundly up-regulated by Ang II in a concentration-dependent manner, which became apparent at $0.1 \mu \mathrm{M}$, with a peak at $10 \mu \mathrm{M}$. 
Fig. 5. Neovascular-related functions of EPCs treated with RhoA/ ROCK signaling inhibitors. (A): Adhesion capacity of EPCs treated with RhoA/ROCK signaling inhibitors. Adhesion capacity of EPCs was not affected by C3 exoenzyme, GGTI-286 and Y-27632. (B): Migration of EPCs cocultured with RhoA/ ROCK signaling inhibitors. Migratory ability of EPCs was not affected by C3 exoenzyme, GGTI-286 and Y-27632. (C): Effect of RhoA/ ROCK signaling inhibitors on EPC in vitro vasculogenesis. EPC in vitro vasculogenesis function was not affected by C3 exoenzyme, GGTI-286 and Y-27632.

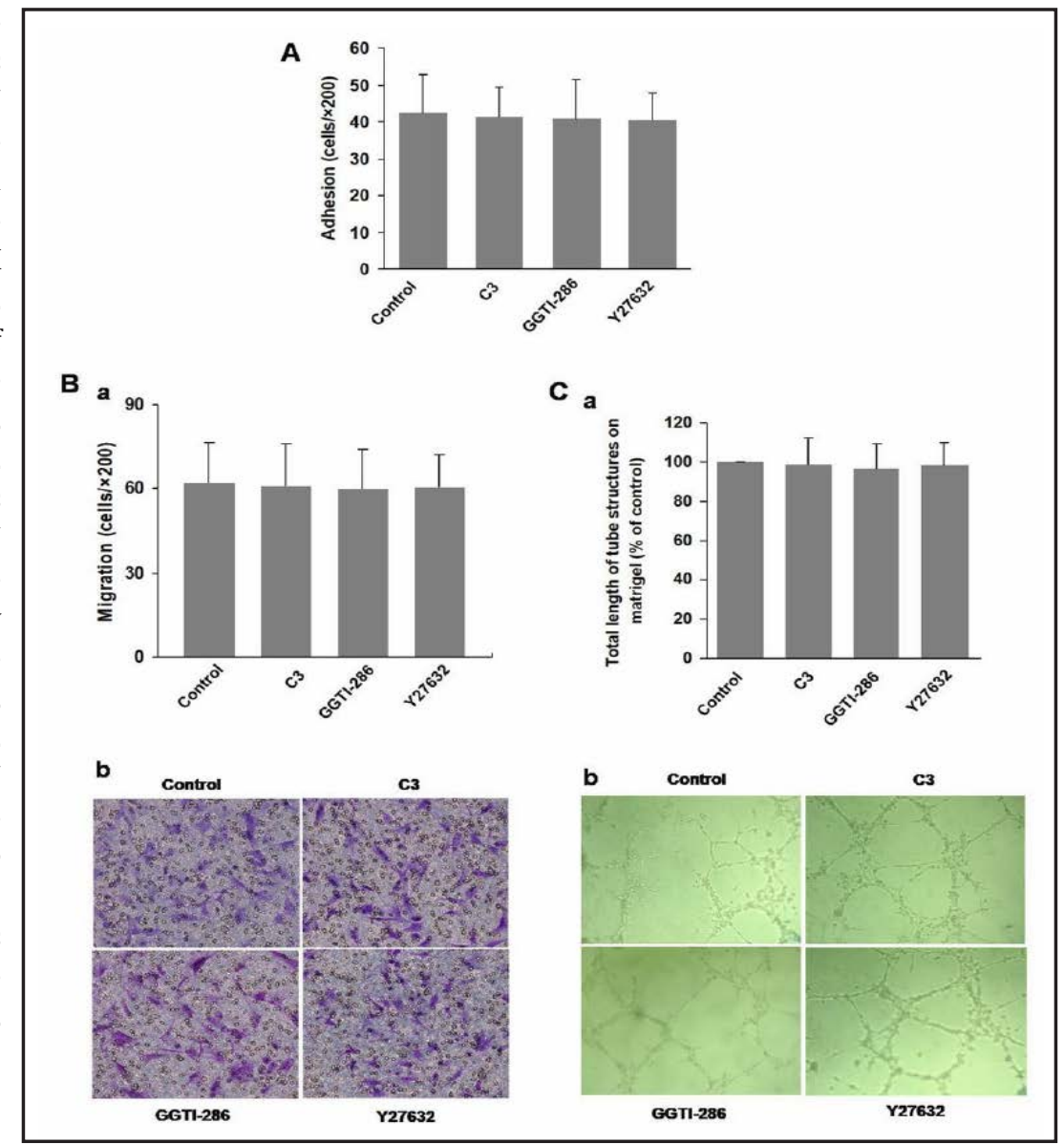

Fig. 6. Effect of RhoA suppression on the neovascular-related functions of EPCs induced by Ang II. shRNA was used to silence RhoA gene expression. RhoA expression and EPC migration, adhesion and in vitro vasculogenesis capacities were investigated. A, RBD-RhoA proteins (upper panel) were detected by Western blot using monoclonal antibody against RhoA. $\beta$-actin was the endogenous control for each sample (lower panel). Values shown are representative of three independent experiments. B-D, EPC migration, adhesion and in vitro vasculogenesis capacities were enhanced by Ang II and these effects could be reversed by RhoA suppression. Data are presented as mean $\pm S D$, \& $\mathrm{P}$ $<0.05$ vs. control group; ${ }^{*} \mathrm{P}<0.01$ vs. control group; $* \mathrm{P}<0.05$ vs. control shRNA group; ** $\mathrm{P}<0.01$ vs. control shRNA group.

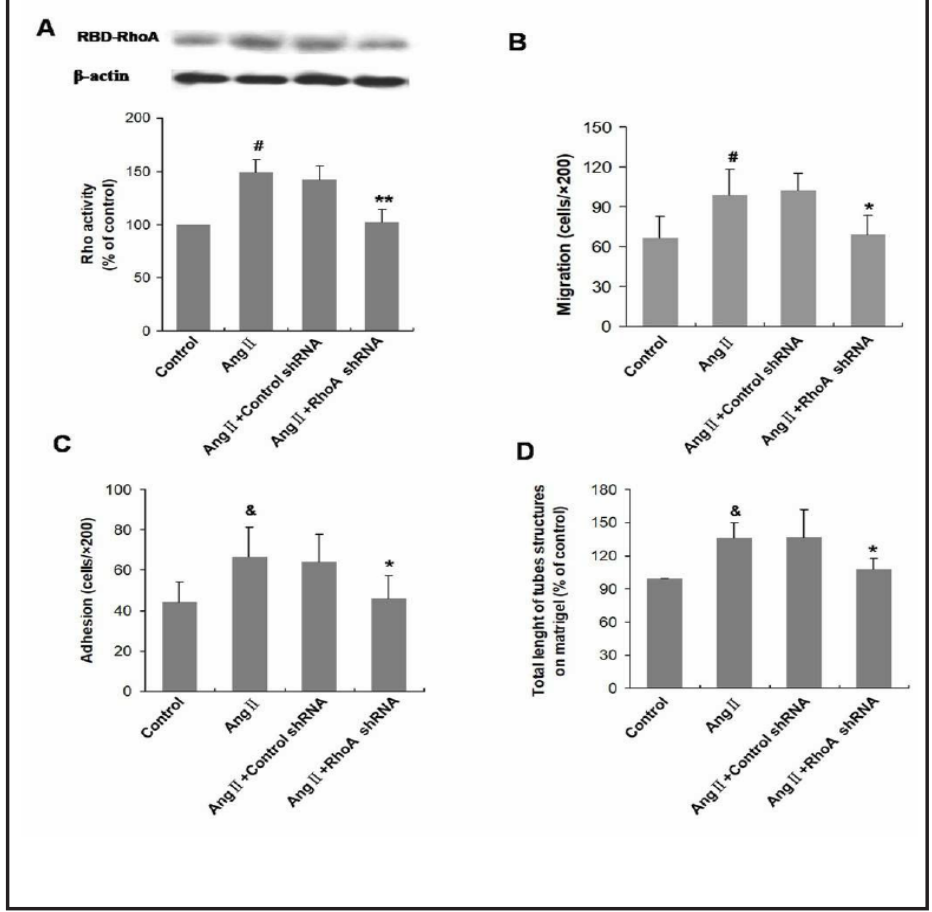


The role of Ang II in EPC functions has been evaluated, and the results of these studies are inconsistent. Endtmann et al. found that the rate of EPC proliferation is not modulated by Ang II (in the presence of $1 \mu \mathrm{mol} / \mathrm{L}$ Ang II for 7 days) [11]. It was also found that in blood MNCs of healthy human volunteers [12], and blood MNCs and bone marrow of rabbits [13], Ang II alone has no effect on the proliferation of EPCs. Ang II was found to significantly promote EPC adhesion, and have no effect on EPC proliferation [14]. It was showed that a subhypertensive dose of Ang II (0.3 mg/kg per day for 28 days) increases ischemia-induced angiogenesis in operatively induced hindlimb ischemia [15]. De Resende et al. also found that Ang II infusion ( $3 \mathrm{ng} / \mathrm{kg} / \mathrm{min}$ for 7 days) enhances the ability of rat ECs to induce tube formation in vitro [16]. These results are consistent with our findings. Meanwhile, Endtmann et al. found that the migration of EPCs is impaired by Ang II $(0.7 \mathrm{mg} / \mathrm{kg}$ per day for 12 days) [11]. Moreover, it was observed that Ang II diminishes the angiogenic function of EPCs in ischemic hind leg [17]. It was found that the outcomes depend on the degree and locus of RAS activation [10]. It appears that the short term RAS activation enhances EPC repair function, however, chronic Ang II stimulation may result in senescence [18].

In this paper, we further explored the underlying mechanism of the effects of Ang II on EPC neovascular-related functions. Recent proof has accumulated that the Rho proteins play an important role in angiogenesis [19]. The Rho proteins, whose most studied member is RhoA, are molecular switches cycling between an inactive guanosine diphosphate (GDP)bound and an active guanosine triphosphate (GTP)-bound state [20]. The most extensively studied RhoA effectors are the serine/threonine Rho-associated kinases (ROCKs), which exist in cytoplasm in autoinhibited state that is disrupted and transported to the plasma membrane upon connection with active RhoA [21]. Recently, a study showed that the RhoA/ ROCK pathway is required for EC capillary formation [22]. Specifically, Hoang et al. found that regulation of RhoA activity can be used either to inhibit or promote the formation of new blood vessels [23]. Dominant negative N19RhoA selectively decreases assembly of ECs into new blood vessels, and in contrast, dominant active V14RhoA enhances ECs to form blood vessels [23]. EC tube formation and membrane localization of RhoA were found to be suppressed by simvastatin, which could be reversed by geranylgeranyl pyrophosphate, the substrate for the geranylgeranylation of RhoA [24]. Moreover, tube formation is inhibited by C3 exotoxin, which inactivates RhoA; by GGTI, a specific inhibitor of the geranylgeranylation of Rho; and by the dominant-negative RhoA. Dominant-active RhoA reverses the effect of simvastatin on EC tube formation [24]. Bryan et al. provided results suggesting that blocking RhoA inhibits VEGF-stimulated angiogenesis [25].

In present study, it was showed that elevated EPC adhesion, migration, and in vitro tube formation stimulated by Ang II could be abolished by C3 exoenzyme, GGTI-286 and Y-27632. Our results suggest that suppression of RhoA/ROCK signaling pathway may inhibit EPC neovascular-related functions. It was also found that the active form of RhoA (GTPbound) was up-regulated after the addition of Ang II and this effect could be reversed by C3 exoenzyme pretreatment. In previous study, it was demonstrated that the amount of active form of Rho is increased by treating cells with LPA, and abolished by C3 exoenzyme treatment in NIH 3T3 cells [26]. Our data and previous findings demonstrate that RhoA/ ROCK signaling is an important mediator in a number of angiogenic processes, and indicate that RhoA/ROCK inhibition would be an efficacious therapeutic strategy for the treatment of neovascular-related disorders.

The direct relationship between these findings was further confirmed by the use of shRNA to silence RhoA gene expression. The data showed that the expression of active form of RhoA was decreased markedly in RhoA shRNA interference group compared with control shRNA interference group. Meanwhile, RhoA silencing resulted in a notable inhibition of EPC migration, adhesion and in vitro vasculogenesis capacities. These results suggested that RhoA activation played a pivotal role in Ang II angiogenic effect.

RhoA/ROCK has been indicated as an upstream regulator of MAPK family members including extracellular signal-regulated protein kinase (ERK), c-Jun-NH2 kinase (JNK), and p38 MAPK (p38) [27-29]. In addition, several studies have shown that MAPK pathway is 
involved in angiogenesis [30]. Meanwhile, Ang II was found to activate MAPK and through this signal pathway elicite many cellular responses [31]. So, it was imperative that we further explored this signaling pathway, as it may be served as an excellent therapeutic target for diseases of aberrant angiogenesis. In previous studies, it was found that RhoA stimulates JNK expression through ROCK by activating JNK [27]. Ang II also was found to elevate active RhoA levels and induce phosphorylation of p38 MAPK [28]. Meanwhile, it was revealed that Ang II increases phosphorylation of JNK, and phosphorylation of p38 MAPK [11]. In this study, we found that phosphorylation of p38 and JNK was elevated by Ang II and attenuated by C3 exoenzyme, GGTI-286, and Y-27632. Ang II had no obvious influence on Erk1/2 phosphorylation. In order to investigate the role of p-38 and JNK signaling pathway on the neovascular-related functions of EPCs induced by Ang II, p38 inhibitor SB202190 and JNK inhibitor SP600125 were used in the study. It was found that the phosphorylation of p-38 and JNK could be significantly reduced by SB202190 and SP600125 respectively. The enhancing effects of Ang II on EPC adhesion, migration and in vitro vasculogenesis could be effectively reversed by SB202190 and SP600125. The results suggest the involvement of p-38 and JNK signaling pathway in the role of Ang II effects on EPCs.

It should be noted that in vivo study using a model of hindlimb ischemia should be performed to further confirm the role of RhoA/ROCK and MAPK signaling pathway in EPC neovascular-related functions. Furthermore, it should be examined whether inhibition of Ang II -induced EPC adhesion, migration and in vitro vasculogenesis by RhoA/ROCK signaling inhibitors can be mimicked by negative mutants of RhoA, or can be reversed by active mutants of p38 and JNK.

\section{Conclusion}

Our data showed that Ang II enhanced EPC neovascular-related functions including migration, adhesion, and in vitro tube formation through activating RhoA/ROCK and MAPK signaling pathway. This study highlights the importance of the RhoA/ROCK and MAPK signaling pathway as a target for anti-angiogenic therapeutic agents.

\section{Acknowledgments}

This work was supported by research grants from the National Natural Science Foundation of China [grant numbers NSFC 81400192 and 81170242], Natural Science Foundation of Zhejiang Province [grant number LQ14H020006], and department of science and technology, Zhejiang Province [grant number 2015C33212].

These authors take responsibility for all aspects of the reliability and freedom from bias of the data presented and their discussed interpretation.

\section{Disclosures}

Conflict of interest: None declared.

\section{References}

1 Folkman J: Angiogenesis in cancer, vascular, rheumatoid and other disease. Nat Med 1995;1:27-31.

2 Yang JX, Chen B, Pan YY, Han J, Chen F, Hu SJ: Zoledronate attenuates angiogenic effects of angiotensin II -stimulated endothelial progenitor cells via rhoa and mapk signaling. PLoS One 2012;7:e46511.

- 3 Freedman SB, Isner JM: Therapeutic angiogenesis for ischemic cardiovascular disease. J Mol Cell Cardiol 2001;33:379-393. 


\section{Cellular Physiology Cell Physiol Biochem 2018;47:2498-2510 \begin{tabular}{l|l} 
DOI: 10.1159/000491623 & $\begin{array}{l}\text { O 2018 The Author(s). Published by S. Karger AG, Basel } \\
\text { www.karger.com/cpb }\end{array}$
\end{tabular}}

Yang et al.:The Role of RhoA in Neovascular-Related Functions of EPCs

4 Asahara T, Murohara T, Sullivan A, Silver M, van der Zee R, Li T, Witzenbichler B, Schatteman G, Isner JM: Isolation of putative progenitor endothelial cells for angiogenesis. Science 1997;275:964-967.

5 Bleiziffer O, Hammon M, Naschberger E, Lipnik K, Arkudas A, Rath S, Pryymachuk G, Beier JP, Sturzl M, Horch RE, Kneser U: Endothelial progenitor cells are integrated in newly formed capillaries and alter adjacent fibrovascular tissue after subcutaneous implantation in a fibrin matrix. J Cell Mol Med 2011;15:2452-2461.

6 Peti-Peterdi J, Kang JJ, Toma I: Activation of the renal renin-angiotensin system in diabetes--new concepts. Nephrol Dial Transplant 2008;23:3047-3049.

-7 Ichiki T: Role of renin angiotensin system in angiogenesis: It is still elusive. Arterioscler Thromb Vasc Biol 2004;24:622-624.

-8 Yang JX, Pan YY, Ge JH, Chen B, Mao W, Qiu YG, Wang XX: Tanshinone II a attenuates TNK- $\alpha$-induced expression of VCAM-1 and ICAM-1 in endothelial progenitor cells by blocking activation of NF- $\kappa$ B. Cell Physiol Biochem 2016; 40:195-206.

-9 YanYun P, Wang S, Yang J, Chen B, Sun Z, Ye L, Zhu J, Wang X: Interruption of CD40 pathway improves efficacy of transplanted endothelial progenitor cells in monocrotaline induced pulmonary arterial hypertension. Cell Physiol Biochem 2015;36:683-696.

10 Qian C, Schoemaker RG, van Gilst WH, Roks AJ: The role of the renin-angiotensin-aldosterone system in cardiovascular progenitor cell function. Clin Sci (Lond) 2009;116:301-314.

11 Endtmann C, Ebrahimian T, Czech T, Arfa O, Laufs U, Fritz M, Wassmann K, Werner N, Petoumenos V, Nickenig G, Wassmann S: Angiotensin II impairs endothelial progenitor cell number and function in vitro and in vivo: Implications for vascular regeneration. Hypertension 2011;58:394-403.

12 Imanishi T, Hano T, Nishio I: Angiotensin II potentiates vascular endothelial growth factor-induced proliferation and network formation of endothelial progenitor cells. Hypertens Res 2004;27:101-108.

-13 Ohtani K, Egashira K, Ihara Y, Nakano K, Funakoshi K, Zhao G, Sata M, Sunagawa K: Angiotensin II type 1 receptor blockade attenuates in-stent restenosis by inhibiting inflammation and progenitor cells. Hypertension 2006;48:664-670.

14 Yin T, Ma X, Zhao L, Cheng K, Wang H: Angiotensin ii promotes no production, inhibits apoptosis and enhances adhesion potential of bone marrow-derived endothelial progenitor cells. Cell Res 2008;18:792799.

15 Tamarat R, Silvestre JS, Kubis N, Benessiano J, Duriez M, deGasparo M, Henrion D, Levy BI: Endothelial nitric oxide synthase lies downstream from angiotensin II -induced angiogenesis in ischemic hindlimb. Hypertension 2002;39:830-835.

16 de Resende MM, Stodola TJ, Greene AS: Role of the renin angiotensin system on bone marrow-derived stem cell function and its impact on skeletal muscle angiogenesis. Physiol Genomics 2010;42:437-444.

17 You D, Cochain C, Loinard C, Vilar J, Mees B, Duriez M, Levy BI, Silvestre JS: Combination of the angiotensinconverting enzyme inhibitor perindopril and the diuretic indapamide activate postnatal vasculogenesis in spontaneously hypertensive rats. J Pharmacol Exp Ther 2008;325:766-773.

-18 Kobayashi K, Imanishi T, Akasaka T: Endothelial progenitor cell differentiation and senescence in an angiotensin II -infusion rat model. Hypertens Res 2006;29:449-455.

-19 Merajver SD, Usmani SZ: Multifaceted role of Rho proteins in angiogenesis. J Mammary Gland Biol Neoplasia 2005;10:291-298.

20 Bryan BA, D'Amore PA: What tangled webs they weave: Rho-GTPase control of angiogenesis. Cell Mol Life Sci 2007;64:2053-2065.

21 Amano M, Fukata Y, Kaibuchi K: Regulation and functions of Rho-associated kinase. Exp Cell Res 2000;261:44-51.

22 Somlyo AV, Bradshaw D, Ramos S, Murphy C, Myers CE, Somlyo AP: Rho-kinase inhibitor retards migration and in vivo dissemination of human prostate cancer cells. Biochem Biophys Res Commun 2000;269:652659.

-23 Hoang MV, Whelan MC, Senger DR: Rho activity critically and selectively regulates endothelial cell organization during angiogenesis. Proc Natl Acad Sci U S A 2004;101:1874-1879.

24 Park HJ, Kong D, Iruela-Arispe L, Begley U, Tang D, Galper JB: 3-hydroxy-3-methylglutaryl coenzyme A reductase inhibitors interfere with angiogenesis by inhibiting the geranylgeranylation of RhoA. Circ Res 2002;91:143-150. 


\section{Cellular Physiology Cell Physiol Biochem 2018;47:2498-2510

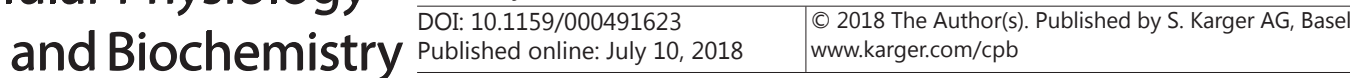

Yang et al.:The Role of RhoA in Neovascular-Related Functions of EPCS

25 Bryan BA, Dennstedt E, Mitchell DC, Walshe TE, Noma K, Loureiro R, Saint-Geniez M, Campaigniac JP, Liao JK, D'Amore PA: RhoA/ROCK signaling is essential for multiple aspects of VEGF-mediated angiogenesis. FASEB J 2010;24:3186-3195.

26 Ren XD, Kiosses WB, Schwartz MA: Regulation of the small GTP-binding protein Rho by cell adhesion and the cytoskeleton. EMBO J 1999;18:578-585.

27 Marinissen MJ, Chiariello M, Tanos T, Bernard O, Narumiya S, Gutkind JS: The small GTP-binding protein RhoA regulates c-jun by a ROCK-JNK signaling axis. Mol Cell 2004;14:29-41.

-28 Shatanawi A, Romero MJ, Iddings JA, Chandra S, Umapathy NS, Verin AD, Caldwell RB, Caldwell RW: Angiotensin III-induced vascular endothelial dysfunction through RhoA/Rho kinase/p38 mitogenactivated protein kinase/arginase pathway. Am J Physiol Cell Physiol 2011;300:C1181-1192.

29 Hong SY, Jeon YM, Lee HJ, Kim JG, Baek JA, Lee JC: Activation of RhoA and FAK induces ERK-mediated osteopontin expression in mechanical force-subjected periodontal ligament fibroblasts. Mol Cell Biochem 2010;335:263-272.

-30 Huang D, Ding Y, Luo WM, Bender S, Qian CN, Kort E, Zhang ZF, VandenBeldt K, Duesbery NS, Resau JH, Teh BT: Inhibition of mapk kinase signaling pathways suppressed renal cell carcinoma growth and angiogenesis in vivo. Cancer Res 2008;68:81-88.

-31 Rodrigues-Diez R, Carvajal-Gonzalez G, Sanchez-Lopez E, Rodriguez-Vita J, Rodrigues Diez R, Selgas R, Ortiz A, Egido J, Mezzano S, Ruiz-Ortega M: Pharmacological modulation of epithelial mesenchymal transition caused by angiotensin II . Role of ROCK and MAPK pathways. Pharm Res 2008;25:2447-2461. 